\title{
Plasma Emission Modifications and Instabilities Induced by the Presence of Growing Dust Particles
}

\author{
Maxime Mikikian, Lénaïc Couëdel, Marjorie Cavarroc, Yves Tessier, and Laïfa Boufendi
}

\begin{abstract}
Formation of dust particles in a plasma can strongly change its properties due to electron attachment on dust surface. An easy way to detect dust formation is to analyze modifications of the plasma emission. In this paper, changes in the plasma emission are related to the growth of dust particles. We particularly show that dust formation induces low-frequency plasma instabilities. Another interesting induced effect is the formation of an enhanced emission region which is dust free and usually named "void."
\end{abstract}

Index Terms-Complex plasma, dust particle growth, dusty plasma, instabilities, oscillations, plasma emission, void.

$\mathbf{P}$ LASMAS ARE widely encountered in nature and industries. In many environments, these plasmas also contain dust particles (i.e., solid bodies from a few nanometers to centimeters) and are thus called dusty (or complex) plasmas. In astrophysics, these media are found in comet tails or planetary atmospheres. In artificial plasmas, these dust particles are fatal for processes in microelectronics (where cleanliness is needed) and for fusion devices like ITER (International Thermonuclear Experimental Reactor). Nevertheless, in nanotechnology, these media are useful in building nanostructured films or nanometersize devices. Dust particles can be produced from reactive gases (silane and methane) or material sputtering injecting molecular precursors or solid bodies in the plasma. Molecular precursors coming from reactive gases or sputtering thus initiate a complex succession of chemical and physical reactions, leading to the growth of dust particles. As these dust particles are growing, they attach plasma electrons, leading to a disturbance of plasma equilibrium when the dust particle density is high [1]-[3].

In this paper, modifications of plasma emission and selfexcited instabilities induced by the growing particles are visually analyzed. These experiments are performed in the PKE-Nefedov reactor [4] where dust particles are grown by sputtering a polymer layer exposed to a low-pressure radiofrequency $(13.56 \mathrm{MHz})$ argon plasma. The capacitively coupled

Manuscript received November 28, 2007; revised February 4, 2008. This work was supported by the CNES under Contract 02/CNES/4800000059.

M. Mikikian, M. Cavarroc, Y. Tessier, and L. Boufendi are with the Groupe de Recherches sur l'Energétique des Milieux Ionisés, UMR6606, CNRS/Université d'Orléans, 45067 Orléans Cedex 2, France (e-mail: maxime. mikikian@univ-orleans.fr).

L. Couëdel is with the Groupe de Recherches sur l'Energétique des Milieux Ionisés, UMR6606, CNRS/Université d'Orléans, 45067 Orléans Cedex 2, France, and also with the School of Physics, A28, The University of Sydney, Sydney, N.S.W. 2006, Australia.

Digital Object Identifier 10.1109/TPS.2008.920946 discharge is maintained by parallel electrodes of $4 \mathrm{~cm}$ in diameter separated by $3 \mathrm{~cm}$. The typical pressure is about $1.4 \mathrm{mbar}$, and the injected power is $3.5 \mathrm{~W}$. Plasma emission is recorded by using a standard color charge-coupled device camera at 25 frames/s. Simultaneously, the discharge current is monitored to identify the growing step and the occurring instability type.

Fig. 1 shows the plasma emission at different instants after plasma ignition. Fig. 1(a) has been taken $5 \mathrm{~s}$ after plasma ignition and is considered to be representative of a dust-free plasma as the growing kinetics is slow in our experimental conditions. This figure is thus our reference for the following discussion. It clearly shows the two presheath regions of enhanced emission and the plasma column. Fig. 1(b) corresponds to the beginning of self-excited instabilities induced by the high density of negatively charged dust particles [5] disturbing the plasma electrical equilibrium. These dust particle growth instabilities [1] are composed of a complex succession of welldefined phases. In Fig. 1(b), the instability is characterized by a striationlike behavior [1], [6], and two slightly brighter regions appear on both sides of the center. In this regime, bright regions appear alternatively in different places of the discharge. On electrical measurements, the instability signature corresponds to regular oscillations of a few tens of hertz. Even if the sampling rate of the camera is not sufficient to finely analyze this oscillation, it gives important information on plasma evolution as a major complement of electrical measurements. After this relatively regular regime, a chaotic phase occurs with bright regions appearing in a more erratic manner. This chaotic regime is partly characterized by a slight increase in the intensity of the bright regions, as shown in Fig. 1(c). This regime can last a few minutes and slowly tends toward a more and more ordered phase described by a single bright region that can be, at this stage, easily linked to a dust-free region called "void." This region, which can appear with or without precursory instability, is related to the competition of an inward electrostatic force and an outward ion drag force acting on dust particles. Once a clear void region exists in a dusty plasma, different kinds of instabilities are observed. In Fig. 1(d), taken around 7 min after plasma ignition, this void rotates around the vertical symmetry axis of the discharge. The void is clearly identifiable slightly on the right-hand side. The dust cloud at this instant is made of a high density of big dust particles (a few hundreds of nanometers). Their influence on the plasma is important and results in a strong increase of the plasma emission as observed in the void and above the presheath regions. The rotation slows down, and the rotation radius decreases [Fig. 1(e)]. Then, 

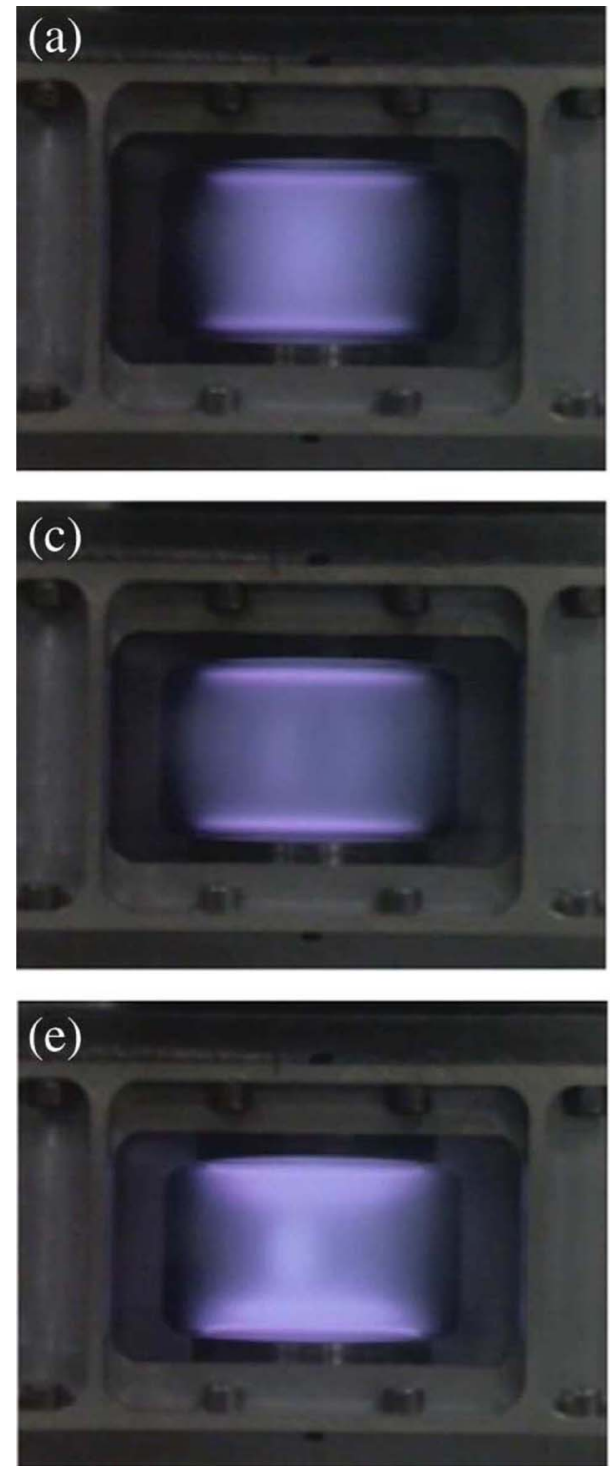
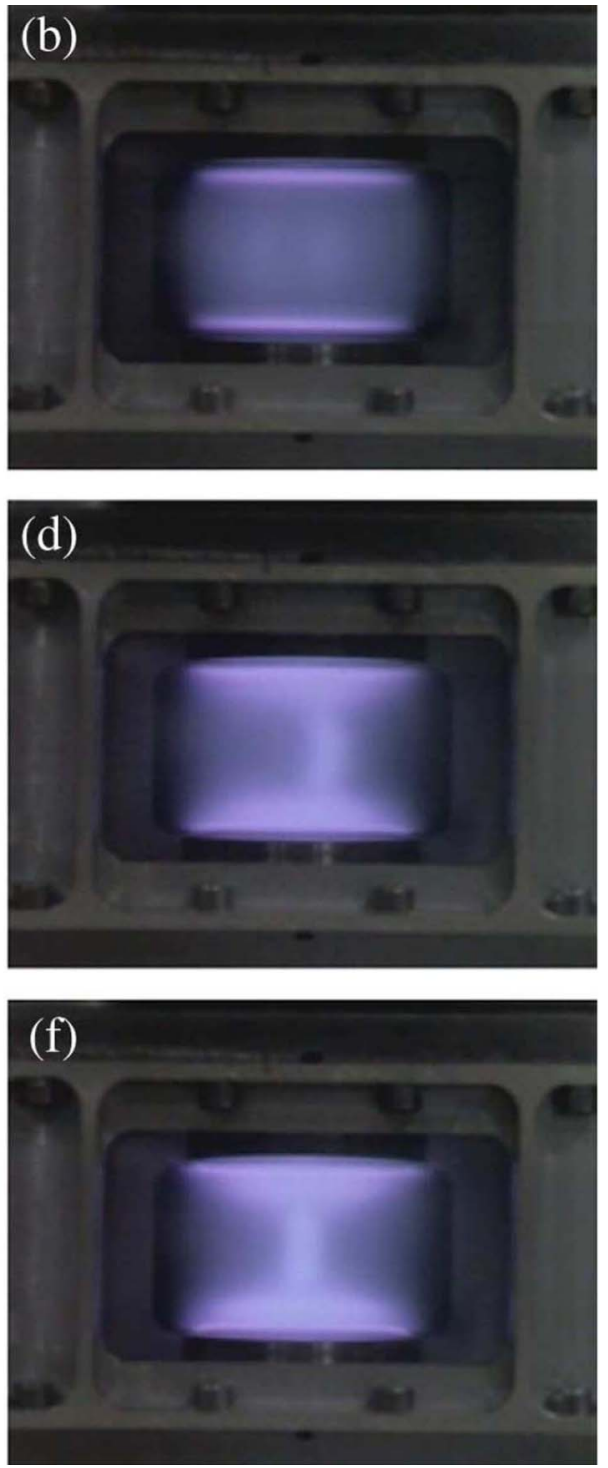

Fig. 1. Plasma emission evolution induced by the presence of dust particles: (a) $5 \mathrm{~s}$ after ignition (AI): No dust particles; (b) 1 min AI: Beginning of instabilities (striationlike) induced by the growing dust particles; (c) 2 min AI: Instabilities with increasing intensity; (d) 7 min AI: Plasma emission enhancement due to the presence of bigger dust particles; instabilities consist of a single enhanced emission region (void) rotating around the vertical axis of symmetry; (e) 15 min AI: Plasma emission enhancement and less off-centered rotation; and (f) 16 min AI: Centered void region with oscillating size.

the enhanced emission void region stabilizes its position in the discharge center. At this time, another type of instability is able to disturb the plasma and the dust cloud. It consists of successive contraction and expansion of the void size [7] [Fig. 1(f)]. Due to its characteristic behavior, this instability has been named the "heartbeat" instability.

In this paper, we presented an easy visual indicator of the presence of a high dust particle density in a plasma. Changes in plasma emission intensity and appearance of selfexcited instabilities easily detected by cameras are consequences of the drastic loss of free electrons to the dust particle surface.

\section{ACKNOWLEDGMENT}

The PKE-Nefedov chamber was made available by the MaxPlanck-Institute for Extraterrestrial Physics, Germany, under the funding of DLR/BMBF under Grants No. 50WM9852.

\section{REFERENCES}

[1] M. Mikikian, M. Cavarroc, L. Couëdel, and L. Boufendi, "Low frequency instabilities during dust particle growth in a radio-frequency plasma," Phys. Plasmas, vol. 13, no. 9, p. 092 103, Sep. 2006.

[2] M. Cavarroc, M. C. Jouanny, K. Radouane, M. Mikikian, and L. Boufendi, "Self-excited instability occurring during the nanoparticle formation in an $\mathrm{Ar}^{-\mathrm{SiH}_{4}}$ low pressure radio frequency plasma," J. Appl. Phys., vol. 99, no. 6, p. $064301,2006$.

[3] M. Cavarroc, M. Mikikian, G. Perrier, and L. Boufendi, "Single-crystal silicon nanoparticles: An instability to check their synthesis," Appl. Phys. Lett., vol. 89, no. 1, p. 013 107, 2006.

[4] A. P. Nefedov et al., "PKE-Nefedov: Plasma crystal experiments on the international space station," New J. Phys., vol. 5, no. 3, pp. 33.1-33.10, 2003.

[5] L. Couëdel, M. Mikikian, L. Boufendi, and A. A. Samarian, "Residual dust charges in discharge afterglow," Phys. Rev. E, Stat. Phys. Plasmas Fluids Relat. Interdiscip. Top., vol. 74, no. 2, p. $026403,2006$.

[6] G. Praburam and J. Goree, "Experimental observation of very lowfrequency macroscopic modes in a dusty plasma," Phys. Plasmas, vol. 3 , no. 4, pp. 1212-1219, Apr. 1996.

[7] M. Mikikian, L. Couëdel, M. Cavarroc, Y. Tessier, and L. Boufendi, "Selfexcited void instability in dusty plasmas: Plasma and dust cloud dynamics during the heartbeat instability," New J. Phys., vol. 9, no. 8, p. 268, 2007. 